\title{
A Blind Multiple Watermarks based on Human Visual Characteristics
}

Ferda Ernawan, Siau-Chuin Liew, Zuriani Mustaffa, Kohbalan Moorthy

Faculty of Computer Systems \& Software Engineering, Universiti Malaysia Pahang, Malaysia

\begin{tabular}{l}
\hline \hline Article Info \\
\hline Article history: \\
Received Apr 9, 2018 \\
Revised Jun 20, 2018 \\
Accepted Jul 11, 2018 \\
\hline
\end{tabular}

\section{Keyword:}

Arnold scrambling

Human visual characteristic

Image watermarking

Multiple watermarks

Singular value decomposition

\begin{abstract}
Digital watermarking is an alternative solution to prevent unauthorized duplication, distribution and breach of ownership right. This paper proposes a watermarking scheme for multiple watermarks embedding. The embedding of multiple watermarks use a block-based scheme based on human visual characteristics. A threshold is used to determine the watermark values by modifying first column of the orthogonal $U$ matrix obtained from Singular Value Decomposition (SVD). The tradeoff between normalize crosscorrelation and imperceptibility of watermarked image from quantization steps was used to achieve an optimal threshold value. The results show that our proposed multiple watermarks scheme exhibit robustness against signal processing attacks. The proposed scheme demonstrates that the watermark recovery from chrominance blue was resistant against different types of attacks.
\end{abstract}

Copyright @ 2018 Institute of Advanced Engineering and Science. All rights reserved.

\section{Corresponding Author:}

Ferda Ernawan,

Faculty of Computer Systems \& Software Engineering,

Universiti Malaysia Pahang,

Lebuhraya Tun Razak 26300 Gambang Kuantan, Pahang, Malaysia.

Email: ferda@ump.edu.my

\section{INTRODUCTION}

Nowadays, digital images can easily be duplicated, copied, distributed and modified. Thus, copyright protection method has a growing demand to ensure the content ownership. Digital watermarking has greatly facilitated to protect the copyright, security, editing of digital data and replication of digital data in the last few decades [1]-[3]. In recent year, multiple watermarks concept of single watermark model drew widespread attention for multimedia security. Multiple watermarking models may contain more than a watermark in the host image [4]. For example, in the case of movie production, multiple originators: director, producer and house production are involved, therefore they need multiple ownership watermarks. For digital image photography, photographing editing and producing digital images also require multiple ownership copyrights. Medical images need multiple watermarks for ownership watermark and alteration verification watermark. For collaborative distributions, the product is embedded by multiple watermarks (for different retailers and distributors).

Many researchers presented the hybrid scheme: Discrete Cosine Transform-Singular Value Decomposition (DCT-SVD) watermarking scheme [5]-[8] that can improve the robustness and invisibility of watermarked images. Lai's scheme [6] revealed the relationship of the orthogonal matrix $U$ in the first column matrix of SVD. The scheme showed an improvement of imperceptibility and robustness under signal processing attacks. Therefore, some SVD-based watermarking techniques explored $U$ or $V$ matrices instead of $S$ as presented in Chang et al. [9], Chung et al. [10], Fan et al. [11], Lai et al. [6]. These techniques avoid the probability of the false positive problems which may occur when embedding is performed into singular value $(S)$. Though many watermarking techniques have been widely used for copyright protection, only few 
methods [11]-[14] have been formulated for multiple watermarking scheme. Multiple watermarking scheme provides more security and robustness [16], [17].

This paper describes a hybrid method using DCT-SVD based on human visual characteristics for multiple watermarks. Referring to [18], red color contributes $65 \%$ cones which sensitive to human eyes, green color provides $33 \%$ sensitivity and blue color has produces $2 \%$ sensitivity. Embedding multiple watermarks on green and blue colors successively can achieve transparency watermarked image. While the watermark can easily be removed when the watermarked image was compressed by JPEG. Luminance and chrominance blue exihibite less sensitivity to human eyes. Therefore, watermark bits are embedded into luminance and chrominance blue components. Embedding of multiple watermarks is performed by examining the relationship of $U_{3,1}$ and $U_{4,1}$ coefficients of SVD. To enhance the security of watermarked images, the two watermarks are scrambled by Arnold chaotic. Finally, the selected blocks are inversed by SVD and DCT to get the watermarked image. The proposed scheme can achieve an improved robustness and imperceptibility of watermarked image.

The related works demonstrate that multiple watermarks are a vital role in multimedia security. This watermarking model can be improved by the hybrid techniques and extra security can be achieved using scrambled watermarks. A new hybrid block-based image watermarking is proposed based on the HVS characteristics and the embedding process is carried out based on modifying first column of orthogonal matrix $U$ of SVD. This scheme attains high robustness against attacks. The highlights and some special features of the proposed scheme are provided as follows:

a. Our scheme proposes multiple watermarks embedding which considers entropy and edge entropy. This paper proposes an optimal threshold for multiple watermarking in luminance and chrominance blue. Our scheme produces minimum distortion in the visual watermarked image.

b. Multiple watermarks embedding are performed by examining the first column of $U$ matrix. Watermark embedding on $U$ matrix of luminance and chrominance blue can improve the robustness and invisibility of multiple watermarks.

c. Confidentially of watermark image is an important information, it should be extracted by authorized users. To improve the security level, multiple watermarks are scrambled before they are embedded into luminance and chrominance blue which can provide extra security in the watermarked image.

d. By finding optimal thresholds for each image component, the quality of the watermarked image produces high image quality and the recovered watermark resistants against different types of attack.

\section{RESEARCH METHOD}

\subsection{Arnold scrambling}

Watermark images are scrambled by Arnold chaotic map to increase the security of multiple watermarking. Scrambled watermarks cannot be recovered without a secret key even attackers successfully extract the watermark from luminance and chrominance blue components of the watermarked image. Arnold scrambling transformation is defined by [19]:

$$
\left(\begin{array}{l}
x^{\prime} \\
y^{\prime}
\end{array}\right)=\left(\begin{array}{ll}
1 & 1 \\
1 & 2
\end{array}\right)\left(\begin{array}{l}
x \\
y
\end{array}\right) \bmod N
$$

where $\left(\begin{array}{l}x^{\prime} \\ y^{\prime}\end{array}\right)$ represents vector position after shifting, $\left(\begin{array}{l}x \\ y\end{array}\right)$ represents original vector position before shifting and mod denotes the modulus operation after division with $N$. The parameter $N$ represents the period of Arnold scrambling. In this experiment, the number of iteration order $N$ is used as a secret key for scrambling transformation. In order to inverse the watermark image, the inverse Arnold transformation can be defined by:

$$
\left(\begin{array}{l}
x \\
y
\end{array}\right)=\left(\begin{array}{cc}
2 & -1 \\
-1 & 1
\end{array}\right)\left(\begin{array}{l}
x^{\prime} \\
y^{\prime}
\end{array}\right) \bmod N
$$

\subsection{Human visual characteristics}

Human visual characteristics less sensitive against redundancy of image information. It can be described through entropy to determine most redundant image information. Entropy was exploited to select significant embedding region. Entropy are applied to determine embedding locations for multiple watermarks 
image. Embedding certain amount of watermark bits in the luminance and chrominance must be invisible to human eyes. The entropy was used to measure the spatial correlation of neighbor pixels. Entropy of an $N$ state is defined by [20]:

$$
E=-\sum_{i=1}^{N} p_{i} \log _{2}\left(p_{i}\right)
$$

Image edge is an important information of image characteristics. Edge entropy of an image block is considered for embedding regions. Edge entropy is given as follows:

$$
E_{\text {edge }}=-\sum_{i=1}^{N} p_{i} \exp ^{1-p_{i}}
$$

where $p_{i}$ denotes the occurrence probability of $i$-th pixel with $0 \leq p_{i} \leq 1$ and $1-p_{i}$ represents the uncertainty or ignorance of the pixel value. The values obtained from combination between entropy and edge entropy are sorted in ascending order and the lowest value are choosen as embedding regions.

\subsection{DCT}

A true-color host image is transformed into $\mathrm{YCbCr}$ color space. Each component (luminance and chrominance blue) is divided into small blocks, then each block is computed by modified entropy. Selected blocks are transformed by two-dimensional DCT to produce the frequency image signals. The twodimensional DCT matrix $B$ of an input image $A$ is computed by [21]:

$$
B_{p q}=\alpha_{p} \beta_{q} \sum_{m=0}^{M-1} \sum_{n=0}^{N-1} A_{m n} \cos \frac{\pi(2 m+1) p}{2 M} \cos \frac{\pi(2 n+1) q}{2 N},
$$

for $p=0,1,2, \ldots, M-1$ and $q=0,1,2, \ldots, N-1$ where

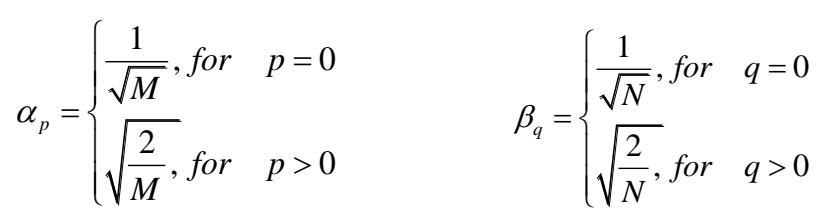

The inverse of two-dimensional DCT is calculated using

$$
A_{p q}=\sum_{m=0}^{M-1} \sum_{n=0}^{N-1} \alpha_{p} \beta_{q} B_{m n} \cos \frac{\pi(2 m+1) p}{2 M} \cos \frac{\pi(2 n+1) q}{2 N}
$$

The DCT coefficients are then transformed by SVD which is described in the next sub-section.

\subsection{SVD}

The SVD factorizes a real or complex matrix into three matrices which are $U, S$ and $V$ matrix. SVD of $A$ can be presented as follows [22]:

$$
A=U S V^{T}
$$

Where $U$ is orthonormal eigenvectors of $A A^{T}, S$ is a diagonal matrix containing the square of the eigenvalues $A$ in descending order and $V$ is orthonormal vectors of $A^{T} A$. Embedding is performed in the first column of the orthogonal matrix $U$ by examining $U_{3,1}$ and $U_{4,1}$ using some rules. The rules are used to embed and extract multiple watermarks in the DCT-SVD domain. The rules are described in the proposed watermarking embedding and extraction algorithms in the next section. 


\subsection{Imperceptibility measurement}

This section describes the metrics to evaluate the proposed watermarking scheme. In order to demonstrate the performance of the proposed scheme, the watermarked imperceptibility is evaluated by structural similarity (SSIM) index. SSIM is computed by:

$$
\operatorname{SSIM}(x, y)=[l(x, y)]^{\alpha} \cdot[c(x, y)]^{\beta} \cdot[s(x, y)]^{\gamma}
$$

where $\alpha>0, \beta>0, \gamma>0$, are parameters which can be adjusted to signify their relative importance.

\subsection{Robustness measurement}

Robustness of watermark extraction is measured by Normalized Cross-Correlation (NC) and Bit Error Rate (BER). NC and BER are given as [23]-[25]:

$$
\begin{gathered}
N C=\frac{\sum_{i=1}^{M} \sum_{j=1}^{N} W(i, j) \cdot W^{*}(i, j)}{\sqrt{\sum_{i=1}^{M} \sum_{j=1}^{N} W(i, j)^{2} \sum_{i=1}^{M} \sum_{j=1}^{N} W^{*}(i, j)^{2}}} \\
B E R=\frac{\sum_{i=1}^{M} \sum_{j=1}^{N} W(i, j) \oplus W^{*}(i, j)}{M \times N}
\end{gathered}
$$

where $\oplus$ denotes the exclusive OR operation. $M$ and $N$ represent rows and columns size of the watermark image, $W^{*}(i, j)$ is the extracted watermark and the $W(i, j)$ is the original watermark.

\section{PROPOSED SCHEME}

\subsection{Watermark insertion}

Watermark insertion process is divided into ten steps. The proposed multiple watermarks scheme is described in Algorithm 1.

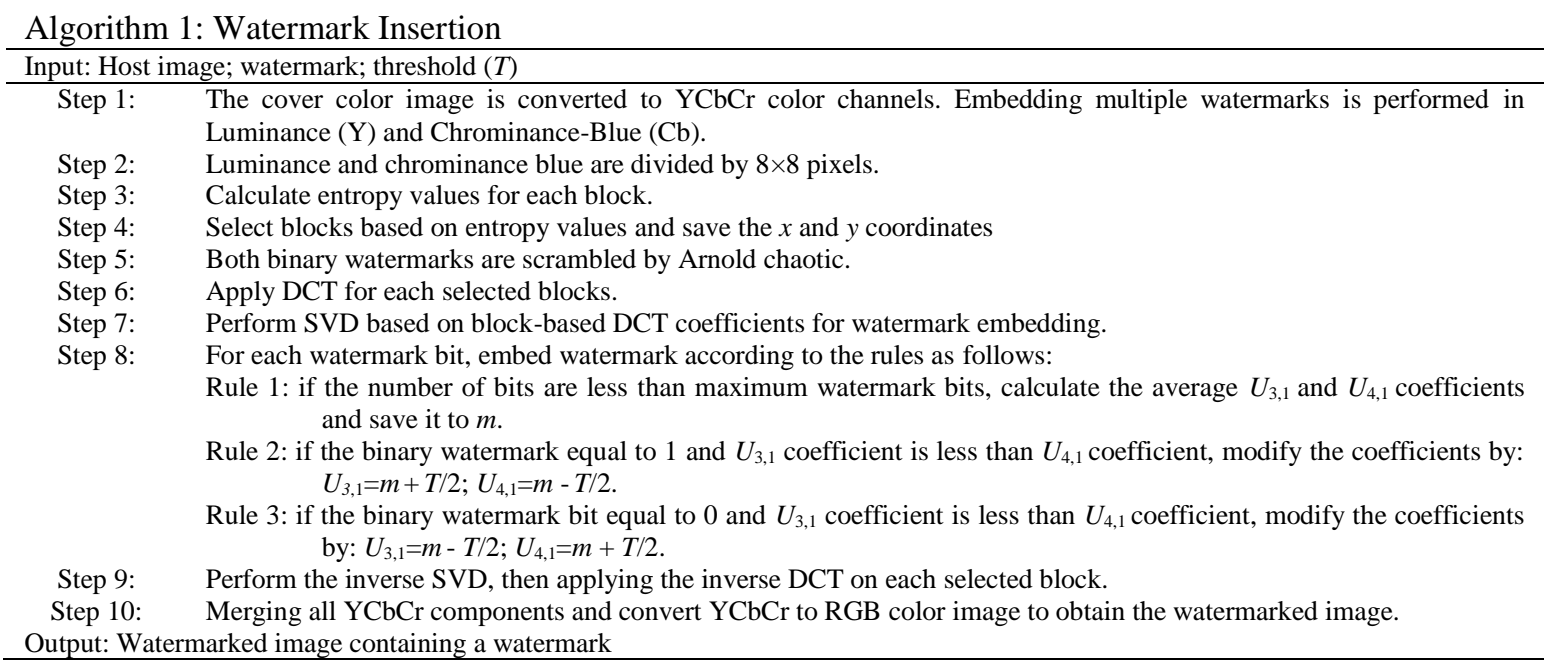

\subsection{Watermark extraction}

Step-by-steps to extract multiple watermarks are divided into sevent steps as described in Algorithm 2. 


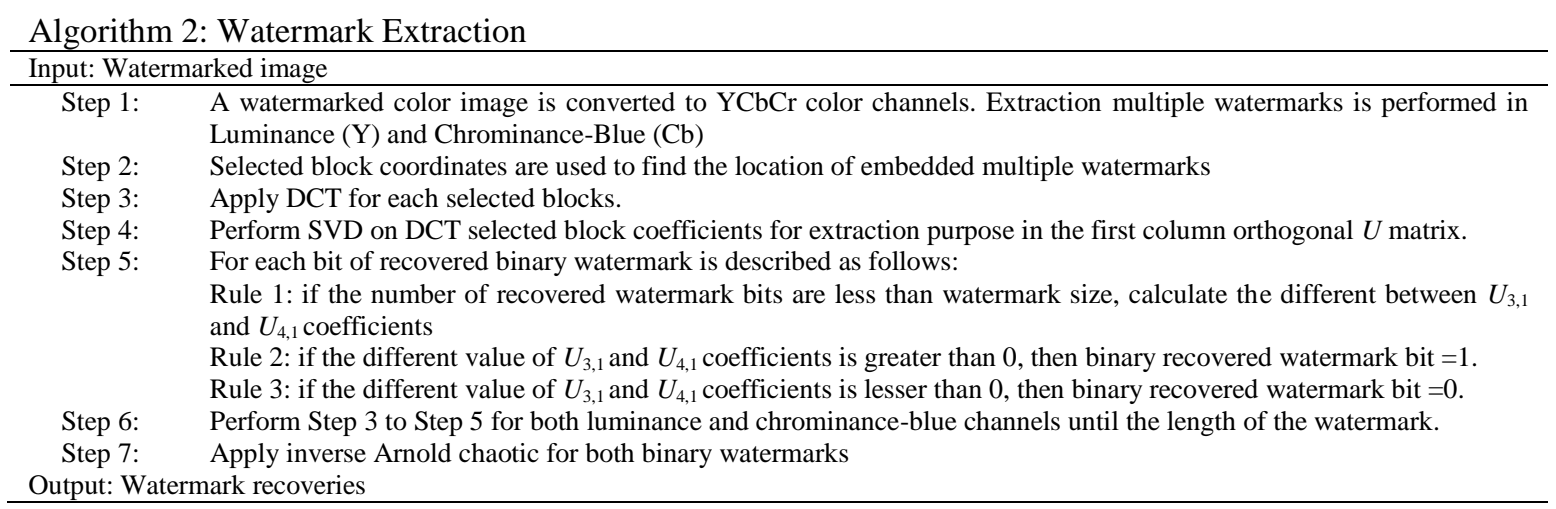

\section{EXPERIMENTAL RESULTS}

The proposed multiple watermarking scheme is employed on five true color images with $512 \times 512$ pixels as shown in Figure 1. The original true color images are taken from CVG-UGR database [26].

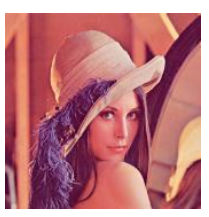

(a)

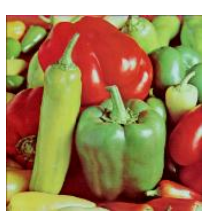

(b)

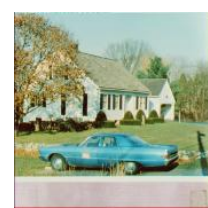

(c)

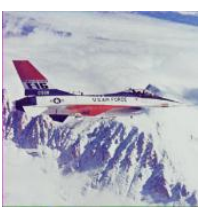

(d)

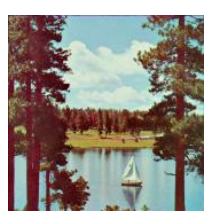

(e)

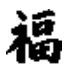

(f)

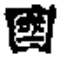

(g)

Figure 1. Host images: (a) Lena, (b) pepper, (c) car, (d) airplane, (e) sailboat (f) first watermark (g) second watermark

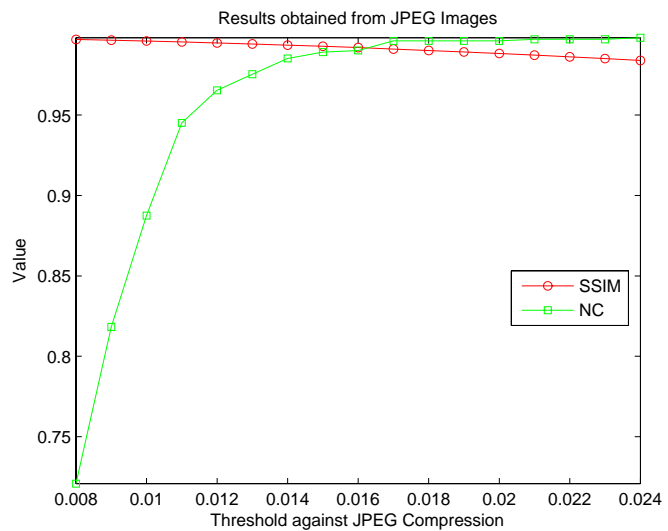

(a)

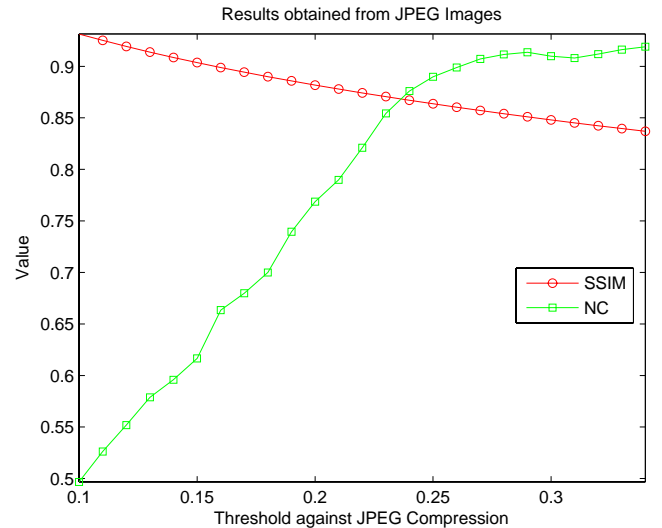

(b)

Figure 2. An optimal threshold for (a) luminance and (b) chrominance blue

The number of selected blocks for luminance and chrominance blue is 1024, it equal to the watermark size with $32 \times 32$ pixels. Using the experiment, we find a threshold as an optimal trade-off between transparency and robustness against JPEG compression for the proposed scheme. JPEG compression is the most popular standard image compression techniques and it has been widely implemented on most digital cameras [27]-[37]. The experimental results have revealed the optimal thresholds as about 0.016 and 0.24 for luminance and chrominance, respectively as shown in Figure 2. The multiple watermark insertion and extraction process are shown in Figure 3 and Figure 4. 


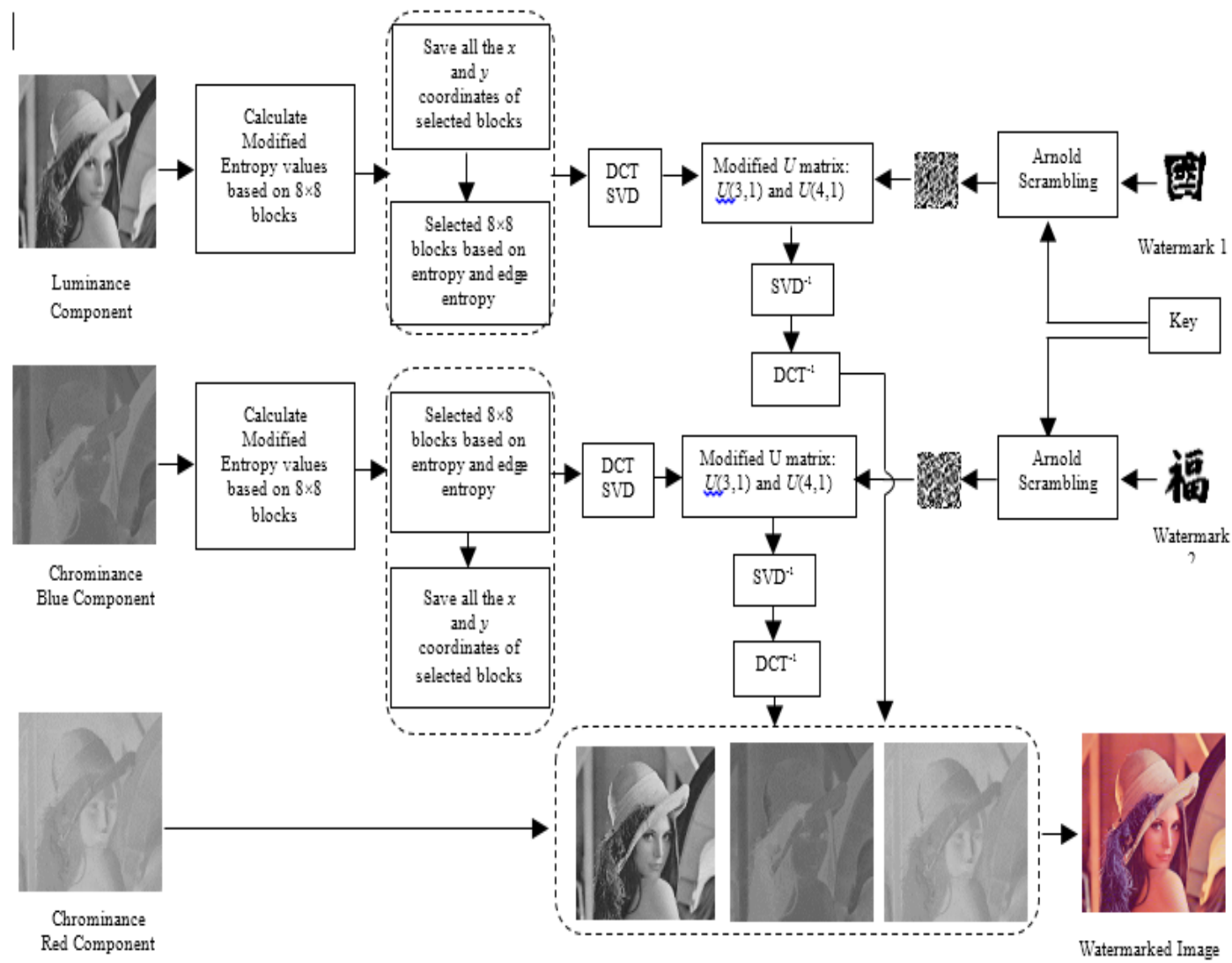

Figure 3. Embedding block diagram

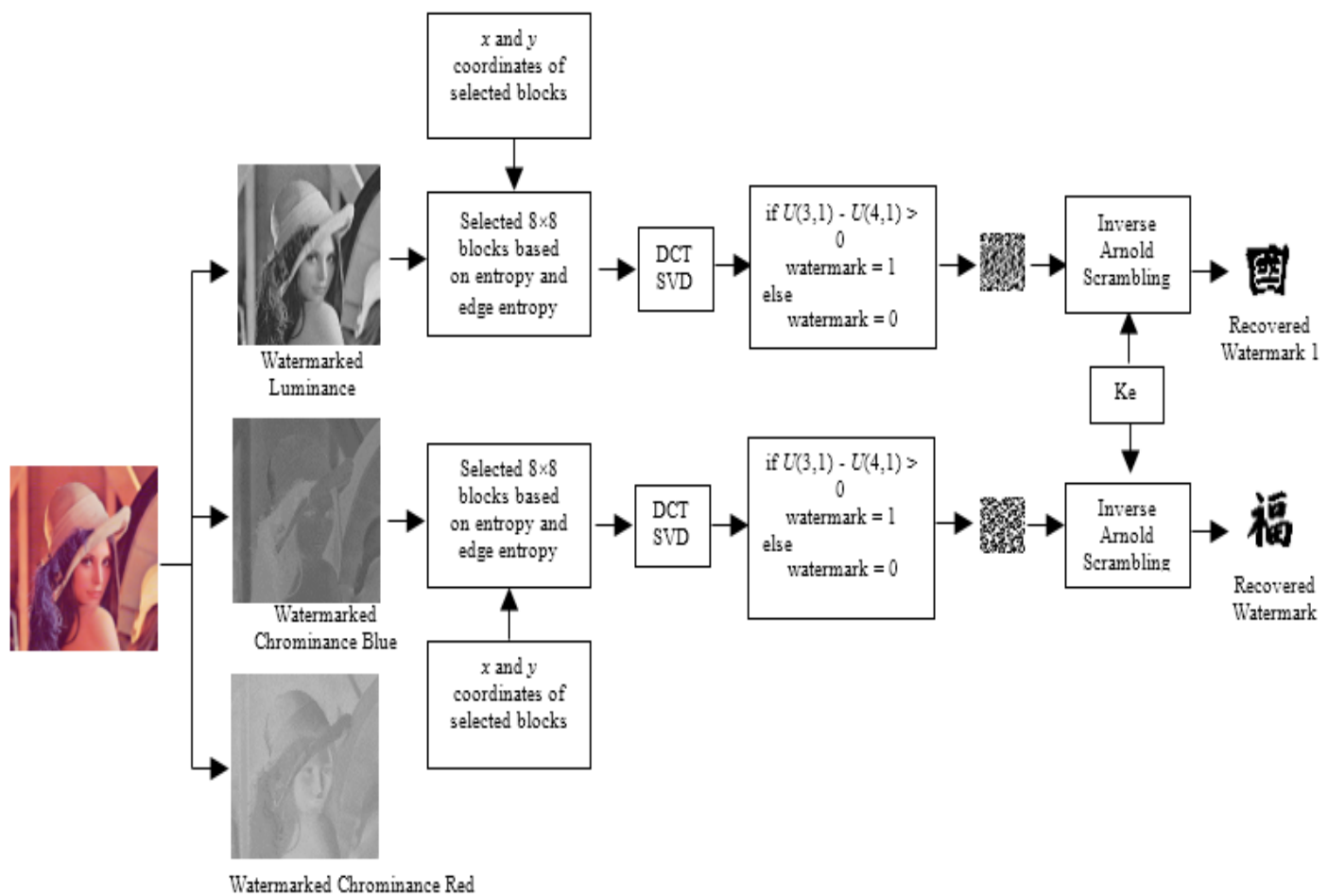

Figure 4. Extraction block diagram 
In this experiment, the proposed multiple watermarks prove the robustness against signal processing attacks especially for embedding in chrominance blue. The embedding in the chrominance blue channel can provide less distortion and it provides higher robustness than embedding in the luminance component. Figure 5 shows the recovered multiple watermarks under the different types of attack. Table 1 and Table 2 show the imperceptibility and robustness of watermarked image from Lena image.

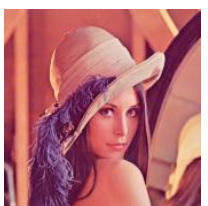

(a)

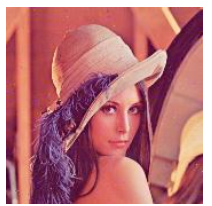

(e)

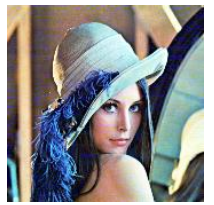

(i)
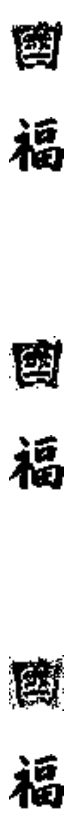

福

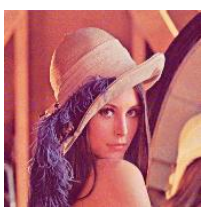

(b)

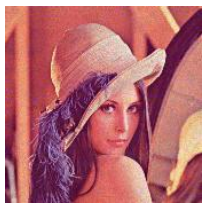

(f)

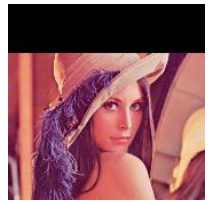

(j)
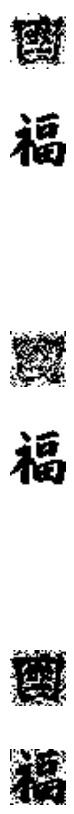

sit

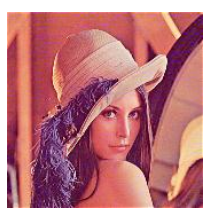

(c)

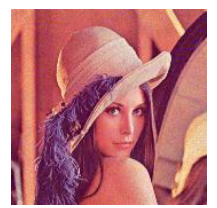

(g)

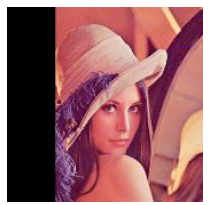

(k)
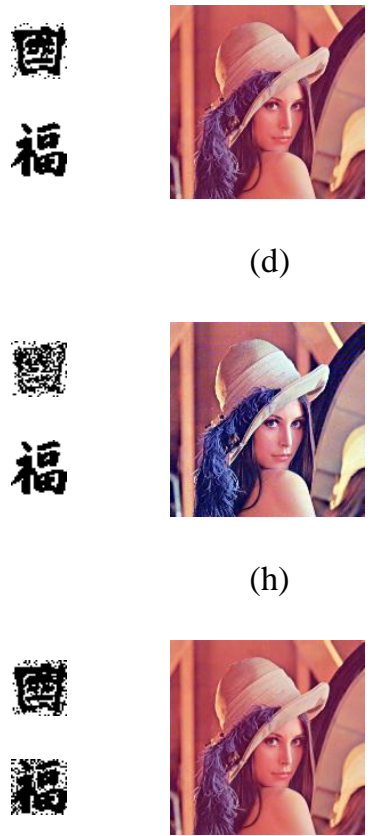

国

酒

(1)

Figure 5. Results under different types of attack and the corresponding recovered watermark using (a) gaussian low pass filter [33], (b) gaussian noise 0.001, (c) sharpening, (d) median filter (3×3), (e) pepper and salt noise $0.1 \%$, (f) speckle noise 0.01 , (g) poisson noise, (h) adjust, (i) histogram equalization attack

(j) cropping rows off $25 \%$, (k) cropping columns off $25 \%$, (l) scaling 0.5

Table 1. NC Values for Lena Image under different Geometrical Attacks

\begin{tabular}{lcccrr}
\multicolumn{1}{c}{ Attack } & \multirow{2}{*}{ SSIM } & \multicolumn{2}{c}{ Watermark 1 } & \multicolumn{2}{c}{ Watermark 2 } \\
& & NC & BER & NC & \multicolumn{1}{c}{ BER } \\
\hline Cropping rows off 25\% & 0.6605 & 0.8100 & 0.1699 & 0.8393 & 0.1475 \\
Cropping rows off 50\% & 0.4364 & 0.6427 & 0.2900 & 0.6471 & 0.2900 \\
Cropping columns off 25\% & 0.6547 & 0.8824 & 0.1094 & 0.8252 & 0.1592 \\
Cropping columns off 50\% & 0.4176 & 0.8293 & 0.1543 & 0.7363 & 0.2285 \\
Rotation 2 $^{\circ}$ & 0.4905 & 0.5542 & 0.4756 & 0.4635 & 0.5078 \\
Rotation 45 $^{\circ}$ & 0.2004 & 0.5069 & 0.4922 & 0.4203 & 0.5186 \\
Translate attack (10, 10) $_{\text {Translate attack (10, 20) }}^{0.2773}$ & 0.5292 & 0.5264 & 0.4650 & 0.5195 \\
Scaling 0.5 & 0.2680 & 0.4063 & 0.4980 & 0.4449 & 0.5146 \\
Scaling 0.25 & 0.8845 & 0.9970 & 0.0029 & 1 & 0 \\
\hline
\end{tabular}

Table 2. NC Values for Lena Image under different Signal Processing Attacks

\begin{tabular}{lcccrr}
\hline \multicolumn{1}{c}{ Attack } & \multirow{2}{*}{ SSIM } & \multicolumn{2}{c}{ Watermark 1 } & \multicolumn{2}{c}{ Watermark 2 } \\
& & NC & BER & NC & BER \\
\hline Gaussian Low Pass Filter [3 3] & 0.8857 & 0.9780 & 0.0225 & 1 & 0 \\
Gaussian Low Pass Filter [5 5] & 0.8881 & 0.9609 & 0.0410 & 1 & 0 \\
Gaussian Noise 0.001 & 0.8039 & 0.9068 & 0.0918 & 1 & 0 \\
Gaussian Noise 0.005 & 0.6512 & 0.7248 & 0.2842 & 1 & 0 \\
Sharpening & 0.7930 & 0.9535 & 0.0449 & 1 & 0 \\
Median Filter [3 3] & 0.8835 & 0.9722 & 0.0283 & 1 & 0 \\
Median Filter [5 5] & 0.9031 & 0.7834 & 0.2813 & 0.9923 & 0.0078 \\
Pepper and Salt Noise 0.1\% & 0.8548 & 0.9831 & 0.0166 & 1 & 0 \\
\hline
\end{tabular}




\begin{tabular}{lcccrr}
\hline \multicolumn{1}{c}{ Attack } & \multirow{2}{*}{ SSIM } & \multicolumn{2}{c}{ Watermark 1 } & \multicolumn{2}{c}{ Watermark 2 } \\
& & NC & BER & NC & BER \\
\hline Pepper and Salt Noise 1\% & 0.7386 & 0.8438 & 0.1533 & 1 & 0 \\
Speckle Noise 0.01 & 0.7079 & 0.7774 & 0.2197 & 1 & 0 \\
Poisson Noise & 0.7466 & 0.8172 & 0.1846 & 1 & 0 \\
Adjust & 0.7925 & 0.9950 & 0.0049 & 1 & 0 \\
Histogram Equalization Attack & 0.6726 & 0.9030 & 0.0957 & 1 & 0 \\
JPEG with Q=40 & 0.8824 & 0.5874 & 0.3857 & 0.7377 & 0.2559 \\
JPEG with Q=50 & 0.8684 & 0.8203 & 0.1729 & 0.8738 & 0.1270 \\
JPEG with Q=60 & 0.8653 & 0.9527 & 0.0469 & 0.9195 & 0.0811 \\
JPEG with Q=70 & 0.8680 & 0.9970 & 0.0029 & 0.9740 & 0.0264 \\
\hline
\end{tabular}

Figure 6 shows bit error rate of the proposed scheme against JPEG and JPEG2000 compression with different types of compression level. It can be noticed that watermark insertion in chrominance blue is more resistants against JPEG2000 than watermark insertion in luminance.

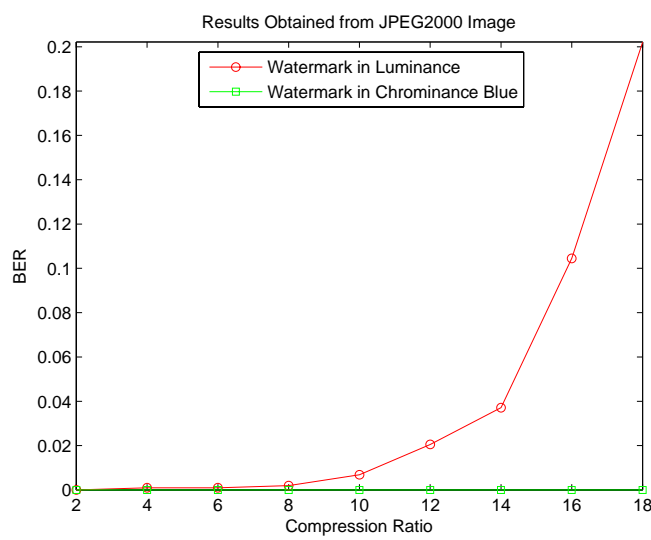

(a)

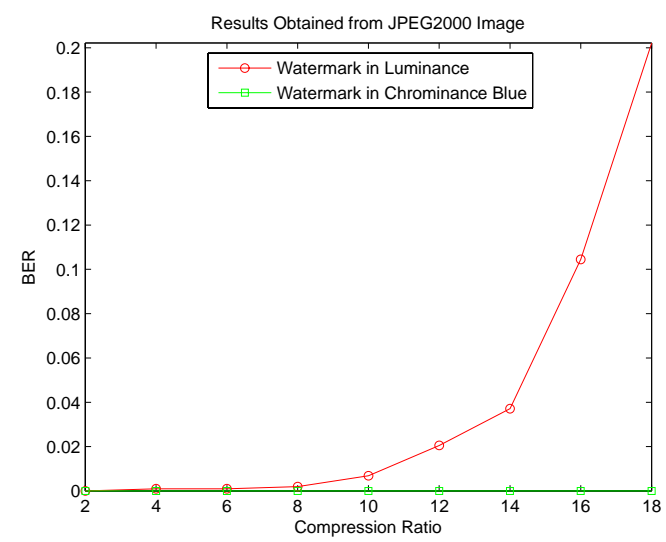

(b)

Figure 6. BER values of the proposed scheme against JPEG and JPEG2000 compression

\section{CONCLUSION}

This paper proposes block-based multiple watermarking scheme based on human visual characteristics. This experiment demonstrated the multiple watermarks insertion into host images by examining $U_{3,1}$ and $U_{4,1}$ of the orthogonal matrix. The proposed scheme provides robustness and resistance against signal processing attacks. The two scrambled watermarks provides extra security and difficult to be identified. The distributed watermarks embedding based on human visual characteristics can achieve high imperceptibility of watermarked image. Furthermore, the embedding scheme for luminance and chrominance blue pairs effectively provides resistance to altered signal processing attacks like JPEG, image noise, image filter, sharpening, and geometric attacks like scaling, translation, cropping. The optimal threshold for multiple watermarks is able to achieve optimal robustness and imperceptibility. The results have proven that our proposed scheme holds excellent robustness and imperceptibility for multiple watermarks.

\section{ACKNOWLEDGEMENTS}

The authors express their thanks to Universiti Malaysia Pahang, Malaysia for providing the financial support for this research project through UMP Research Grant Scheme (RDU170399).

\section{REFERENCES}

[1] F. Ernawan, M. N. Kabir, M. Fadli, Z. Mustaffa, "Block-based Tchebichef image watermarking scheme using psychovisual threshold," 2nd International Conference on Science and Technology Computer, pp. 006-010, 2016.

[2] F. Ernawan, "Robust Image Watermarking Based on Psychovisual Threshold," Journal of ICT Research and Applications, vol.10, no. 3, pp.228-242, 2016.

[3] N.A. Abu, F. Ernawan, N. Suryana, S. Sahib, "Image Watermarking Using Psychovisual Threshold over the Edge," Information and Communication Technology, ICT-EurAsia 2013, LNCS, vol. 7804, pp. 519-527, 2013. 
[4] S. Roy and A.K. Pal, "A blind DCT based color watermarking algorithm for embedding multiple watermarks," International Journal of Electronics and Communications, vol. 72, pp. 149-161, 2017.

[5] H.-T. Hu and L.-Y. Hsu, "Exploring DWT-SVD-DCT feature parameters for robust multiple watermarking against JPEG and JPEG2000 compression," Computers and Electrical Engineering, vol. 41, pp. 052-063, 2015.

[6] C.-C. Lai, "An improved SVD-based watermarking scheme using human visual characteristics," Optics Communications, vol. 284, no. 4, pp. 938-944, 2011

[7] M. Li and C. Han, "A DCT-SVD domain watermarking for color digital image based on compressed sensing theory and chaos theory," Seventh International Symposium on Computational Intelligence and Design (ISCID), pp. 35-38, 2014.

[8] M. Ali, C.W. Ahn, M. Pant, "A robust image watermarking technique using SVD and differential evolution in DCT domain," Optik, vol. 125, pp. 428-434, 2014.

[9] C.C.Chang, P. Tsai, C.C. Lin, "SVD-based digital image watermarking scheme," Pattern Recognition Letter, vol. 26, no. 10, pp. 1577-1586, 2005.

[10] K.L. Chung, W.N. Yang, Y.H. Huang, S.T. Wu, Y.C. Hsu, "On SVD-based watermarking algorithm," Applied Mathematics and Computation, vol. 188, no. 1, pp. 54-57, 2007.

[11] M.Q. Fan, H.X. Wang, S.K. Li, "Restudy on SVD-based watermarking scheme," Applied Mathematics and Computation, vol. 203, no. 3 pp.926-930, 2008.

[12] V. Khanduja, S. Chakraverty and O.P. Verma, "Enabling information recovery with ownership using robust multiple watermarks," Journal of Information Security and Applications, vol. 29, pp. 80-92, 2016.

[13] V. Khanduja, S. Chakraverty and O.P. Verma, R. Tandon, S. Goel, "A robust multiple watermarking technique for information recovery," IEEE International Conference on Advance Computing, pp. 250-255, 2014.

[14] S. Behnia, M. Teshnehlab, P. Ayubi, "Multiple-watermarking scheme based on improved chaotic maps," Communications in Nonlinear Science and Numerical Simulation, vol. 15, no. 9, pp. 2469-2478, 2010.

[15] H.-C. Huang, S.-C. Chu, J.-S. Pan, C.-Y. Huang and B.-Y. Liao, "Tabu search based multi-watermarks embedding algorithm with multiple description coding," Information Sciences, vol. 181, no. 16, pp. 3379-3396, 2011.

[16] N. Mohananthini and G. Yamuna, "Comparison of multiple watermarking techniques using genetic algorithms," Journal of Electrical Systems and Information Technology, vol. 3, no. 1, pp. 68-80, 2016.

[17] R. Chamlawi, A, Khan and I. Usman, "Authentication and recovery of images using multiple watermarks," Computers \& Electrical Engineering, vol. 36, no. 3, pp. 578-584, 2010.

[18] R.C. Gonzalez, R.E.Wood, "Digital image processing, 2nd edition," India: Pearson Education, 2002.

[19] M. Khalili, "DCT-Arnold chaotic based watermarking using JPEG-YCbCr," Optik - International Journal for Light and Electron Optics, vol. 126, no. 3, pp. 4367-4371, 2015.

[20] F. Ernawan, M. Ramalingam, A.S. Sadiq, Z. Mustaffa, "An improved imperceptibility and robustness of 4x4 DCTSVD image watermarking using modified entropy," Journal of Telecommunication, Electronic and Computer Engineering, vol. 9, no. 2-7, pp. 111-116, 2017.

[21] F. Ernawan and S.H. Nugraini, "The optimal quantization matrices for JPEG image compression from psychovisual threshold," Journal of Theoretical and Applied Information Technology, vol. 70, no. 3, pp. 566-572, 2014.

[22] S.-L. Jia, "A novel blind color images watermarking based on SVD," Optik - International Journal for Light and Electron Optics, vol. 125, no. 12, pp. 2868-2874, 2014.

[23] M. Boussif, N. Aloui, A. Cherif, "New Watermarking/Encryption Method for Medical Images Full Protection in mHealth," International Journal of Electrical and Computer Engineering, vol. 7, no. 6, pp. 3385-3394, 2017.

[24] V.A. Kumar, C. Dharmaraj, Ch. S. Rao, "A Hybrid Digital Watermarking Approach Using Wavelets and LSB," International Journal of Electrical and Computer Engineering, vol. 7, no. 5, pp. 2483-2495, 2017.

[25] I. Iwut, G. Budiman, L. Novamizanti, "Optimization of Discrete Cosine Transform-Based Image Watermarking by Genetics Algorithm," International Journal of Electrical and Computer Science, vol. 4, no. 1, pp. 91-103, 2016.

[26] R. Rodriguez-Sanchez, J. Martinez-Baena, A. Garrido, J.A. Garcia, J. Fdez-Valdivia, M.C. Aranda, "Computer Vision Group", University of Granada, 2002, http://decsai.ugr.es/cvg/dbimagenes/c512.php.

[27] F. Ernawan, N. Kabir, K.Z. Zamli, "An efficient image compression technique using tchebichef bit allocation," Optik - International Journal for Light and Electron Optics, vol. 148, pp. 106-119, 2017.

[28] F. Ernawan, N.A. Abu, N. Suryana, "An adaptive JPEG image compression using psychovisual model," Advanced Science Letters, vol. 20, no. 1, pp. 26-31, 2014.

[29] N.A. Abu, F. Ernawan, "A novel psychovisual threshold on large DCT for image compression," The Scientific World Journal, vol. 2015, no. 2015, pp. 001-011, 2015.

[30] N.A. Abu, F. Ernawan, N. Suryana, "A generic psychovisual error threshold for the quantization table generation on JPEG image compression," $9^{\text {th }}$ International Colloquium on Signal Processing and its Applications, pp. 39-43, 2013.

[31] F. Ernawan, M.N. Kabir, J.M. Zain, "Bit allocation strategy based on psychovisual threshold in image compression," Multimedia Tools and Applications, pp. 1-24, 2017.

[32] F. Ernawan, Z. Mustaffa, L.B. Aji, "An efficient image compression using bit allocation based on psychovisual threshold," Information (Japan), vol. 9(9B), pp. 4177-4182, 2016.

[33] N.A. Abu, F. Ernawan, "Psychovisual threshold on large tchebichef moment for image compression," Applied Mathematical Sciences, vol. 8, no. 140, pp. 6951-6961, 2014.

[34] F. Ernawan, N.A. Abu, N. Suryana, "Adaptive Tchebichef Moment Transform Image Compression Using Psychovisual Model," Journal of Computer Science, vol. 9, no. 6, pp. 716-725, 2013. 
[35] F. Ernawan, N.A. Abu, N. Suryana, "An Optimal Tchebichef Moment Quantization Using Psychovisual Threshold for Image Compression,” Advanced Science Letters, vol. 20, no. 1, pp. 70-74, 2014.

[36] F. Ernawan, N.A. Abu and N. Suryana, "TMT Quantization Table Generation Based on Psychovisual Threshold for Image Compression," International Conference of Information and Communication Technology (ICoICT 2013), pp. 202-207, 2013.

[37] N.A. Abu, F. Ernawan, S. Sahib, "Psychovisual Model on Discrete Orthonormal Transform," International Conference on Mathematical Sciences and Statistics (ICMSS 2013), pp.309-314, 2013. 